\title{
Supervised Public Network Direct Recording Electronic Voting (PNDRE Voting) on Existing Global System for Mobile Communication Infrastructure; a Panacea for Cheap E-voting System Implementation in Nigeria
}

\author{
Nwogu Emeka Reginald ${ }^{1}$, Onwuachu Uzochukwu C. ${ }^{2}$ \\ ${ }^{1}$ Michael Okpara University of Agriculture, Umudike, Umuahia, Abia state, Nigeria \\ ${ }^{2}$ Imo Sate Univesity, Owerri, Imo State, Nigeria \\ 1'nwogu.emeka@gmail.com, ${ }^{2}$ uzochukwuchristian@yahoo.com
}

\begin{abstract}
This work suggests a way of bringing down the cost of acquiring electronic voting in Nigeria by implementing Supervised Public Network Direct Recording Electronic Voting (PNDRE Voting) on the existing Global System for Mobile (GSM) Communication infrastructure in the Country. A review of the telecommunication industry in Nigeria was done. A system that embeds electronic voting in the existing GSM infrastructure was proposed. This system involves a three-level network setup that includes the public network (hosts electronic voting terminals), the Demilitarized Zone (hosts the forwarding server) and the Secure Local Area Network (hosts the electoral commission's private servers). A comparative analysis of the current system together with the proposed system was done with keen interest in the cost of implementation. It was established that this system ensures reduced cost of implementation with good cost saving; thereby encouraging massive acceptance and deployment especially in countries with well developed GSM infrastructure. The system is also proposed with good security architecture.
\end{abstract}

Keywords: GSM, E-voting, PNDRE, Voting Terminal, Tallying Server

\section{INTRODUCTION}

VoteHere Inc (2002) defined an electronic voting (e-voting) system as a system in which the election data is recorded, stored and processed primarily as digital information.

Oostveen .A.M. and Bessdaar P.V. (2009) defined it is an electronic system which uses electronic ballot that allows voters transmit their secure and secret voted ballot to election officials over the computer.

Supervised electronic voting involves voters casting their votes before electoral officials who supervise the voting process. This means voters still have to converge at a polling booth where they can access the e-voting facility in order to cast their voters. This does not necessarily mean the official has to observe voters as they cast their votes.

As Nwogu Emeka Reginald (2015) puts it, So many challenges are associated with elections and electoral processes; these have made it even more difficult for countries that run manual electoral system to effectively organize free and fair elections.

Consequently, most countries have introduced e-voting systems and platforms, in a bid to make the electoral process and system less cumbersome and easy to manage.

Despite all these, there have been strong objections against the introduction of electronic voting in most countries, with several arguments backing the claim that the disadvantages of this technology far more outweigh its advantages. While some opponents of e-voting have backed their argument with the claim that an e-voting system makes high-tech electoral malpractice possible, others have persistently argued that such a system has very high cost of implementation and thus a heavy financial burden on any country wishing to embark on it, thereby discouraging some countries especially the developing countries from embarking on an e-voting project. These claims especially the later may not be unfounded. 
However, pro-technology pundits have consistently countered this claim, arguing strongly that the introduction of verifiable, accountable, auditable and reliable e-voting technology in the electoral process can go a long a way in addressing the challenges of managing a national election. According to Ankit Anand and Pallavi Divya (2012), electronic voting systems provide some characteristics different from the traditional voting technique, and also provide improved features over traditional voting system such as accuracy, convenience, flexibility, privacy, verifiability and mobility.

Regrettably, high cost of implementation continues to plague the acceptance of this technology, especially in the developing countries. This has resulted in continuous search for cheaper but very secure ways of implementing electronic voting, especially with the use of available telecommunication infrastructure.

This work suggests a way of integrating and implementing supervised electronic voting on existing GSM infrastructure, in order to cut down the implementation cost.

\subsection{Scope of the Work}

- This work proposes a way of embedding supervised electronic voting on existing GSM infrastructure, in order to reduce the total cost of acquiring and implementing an electronic voting system

- Emphasis is more on cost savings rather than implementation procedures

- It did not discuss ways of securing this system from attack, it only suggested.

\section{REVIEW OF VOTING TECHNOLOGIES}

R. Mercuri (2002), Het Ne Oo and Aye Moe Aung (2013) and G.O. Ofori-Dwumfuo and E. Paatey (2011) identified the types of voting to include:

\section{Paper-based voting system}

This system uses paper ballot, where a prospective voter would have to either mark the ballot space for their preferred candidate or print their thumb on the space using ink. This type of voting technology requires huge time and human resources. The advantage of this system is that casted ballots can easily be audited by authorized persons or group.

\section{Lever voting system}

In this voting technology, each lever is associated to a particular candidate. The voter pulls the lever to cast their vote for their preferred candidate. This kind of voting machine can count the ballots automatically, but requires considerable user training.

\section{Direct recording electronic (DRE) voting system}

This type of system records votes by means of a ballot display provided on a computer screen and stores such records in a removable memory. These machines do not transmit the cast ballots electronically. The election representative will have to transmit the result by hand carriage to the result collation centre.

\section{Punch card voting system}

Here, voters use metallic hole-punch to punch a hole on a blank ballot. This can count votes automatically, but may give wrong result determination if incomplete perforation is made.

\section{Public network DRE voting systems (PNDRE)}

This is an improvement on the DRE voting system. The difference is that cast ballots are transmitted electronically over a public network to a central server/collation centre. The votes may be transmitted as individual ballots as they are cast, or periodically as batches of ballots, or as one single batch, at the end of voting.

\section{Precinct count voting systems (PCVS)}

This system counts and stores the result in each polling unit electronically and puts it in a tabular form. These results are subsequently transmitted to a central location over public telecommunication networks.

\section{Central count voting systems (CCVS)}

This type of system collates the results from different precincts at a central location. Voted ballots are safely stored temporarily at the polling station. These ballots are then transported or transmitted to a central counting location. 
Supervised Public Network Direct Recording Electronic Voting (PNDRE Voting) on Existing Global System for Mobile Communication Infrastructure; a Panacea for Cheap E-voting System Implementation in Nigeria

\subsection{The Telecommunication Industry In Nigeria}

The telecommunication industry in Nigeria with more than enough voice channel capacity, very robust internet broadband services and good internet gateway facility can be placed in the class of well developed telecommunication industries of the world. The country also has a good mix of Global System for Mobile (GSM) communication networks, Code Division Multiple Access (CDMA) networks and Fixed Land Line networks.

According to Nigerian Communications Commission (2013), Nigeria at the moment has a total of four (4) active GSM service providers that include MTN Nigeria Communications, Globacom Limited, Airtel Nigeria and EMTS Limited (Etisalat). These companies provide the country with telecommunication infrastructure for both voice and data services covering almost the entire landscape of the country.

Nigerian Communication Commission (2014) reports that at the end of December 2013, just about thirteen years after full liberalisation, Nigeria had over 127.6 million active lines with a tele-density of over $91.15 \%$; while the total number of Base Transceiver stations sites increased from 23,105 in December 2012 to 28,289 in December 2013, a sharp increase of $22.44 \%$.

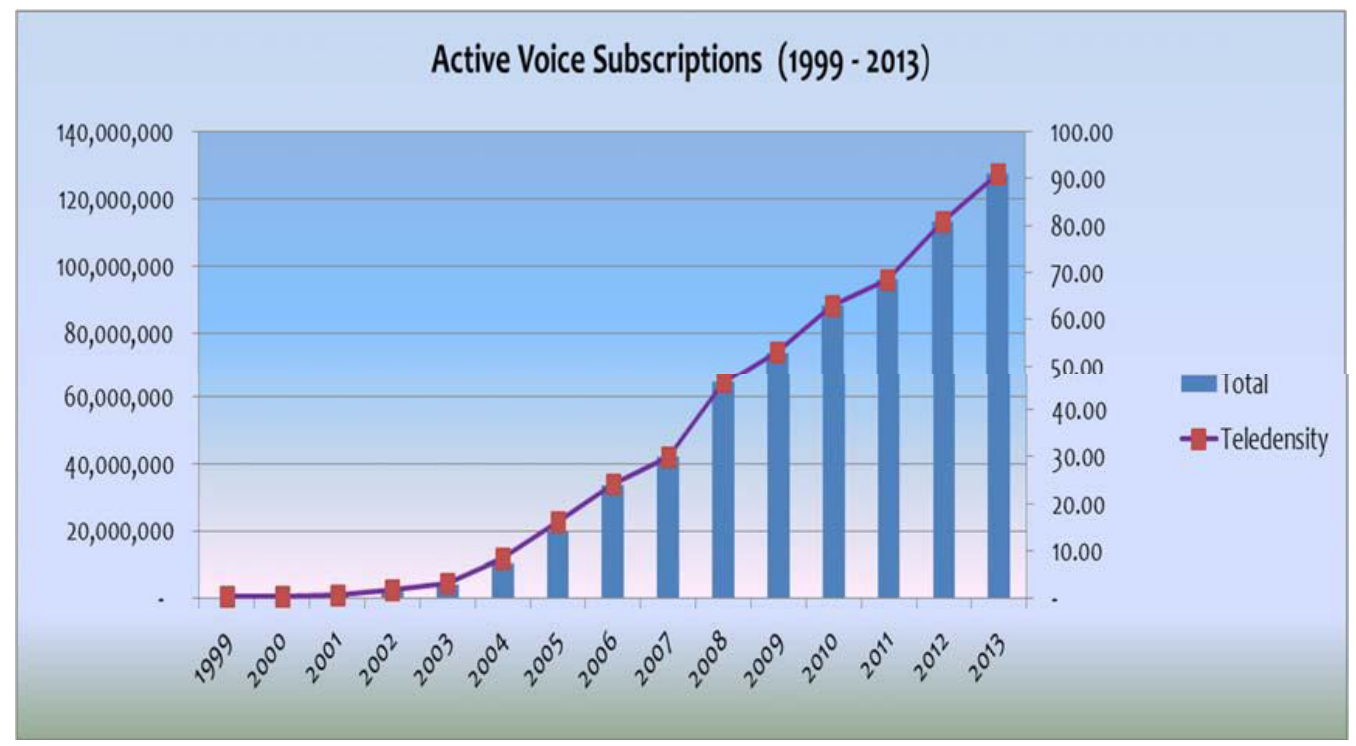

Fig1. Active voice subscriptions (1999 - 2013)

Source: Nigerian Communications Commission (2013).

Table1. Installed Capacities, Active Voice Subscriptions \& Number of Base Stations (GSM) Source: Nigerian Communication Commission (2014)

\begin{tabular}{|l|l|l|l|l|}
\hline $\mathbf{2 0 1 2}$ & MTN & GLO & AIRTEL & EMTS \\
\hline $\begin{array}{l}\text { Installed } \\
\text { Capacity }\end{array}$ & $72,000,000$ & $37,150,107$ & $41,790,000$ & $30,000,000$ \\
\hline $\begin{array}{l}\text { Active Voice } \\
\text { Subscriptions }\end{array}$ & $47,440,991$ & $24,124,716$ & $23,092,195$ & $14,912,801$ \\
\hline $\begin{array}{l}\text { No. of Base } \\
\text { Stations }\end{array}$ & 8,467 & 5,836 & 3,660 & 5,142 \\
\hline $\mathbf{2 0 1 3}$ & MTN & GLO & AIRTEL & EMTS \\
\hline $\begin{array}{l}\text { Installed } \\
\text { Capacity }\end{array}$ & $80,000,000$ & $39,396,740$ & $58,000,000$ & $40,000,000$ \\
\hline $\begin{array}{l}\text { Active Voice } \\
\text { Subscriptions }\end{array}$ & $56,766,085$ & $25,933,867$ & $24,847,567$ & $17,035,276$ \\
\hline $\begin{array}{l}\text { No. of Base } \\
\text { Stations }\end{array}$ & 11,551 & 6,305 & 5,997 & 4,436 \\
\hline
\end{tabular}

*These figures submitted are as at December 31 st of each year (2012 \& 2013) 
Similarly, the NCC in Nigerian Tribune (April 7, 2015) put the number of internet users on the Global System for Mobile Communications (GSM) networks at 81,892,840 in January 2015, an increase from the 76,322,802 users recorded in December 2014.

This data shows how developed the Nigerian GSM industry is, as almost all occupied areas in Nigeria have GSM connectivity.

\subsection{Conventional E-Voting Design}

The most important components of electronic voting are the electronic voting client or terminal (registers and captures votes (ballots) cast by voters), Electronic Voting Tallying Server (receives the ballots from the voting terminal, processes and collates the votes), and the Voter Information Database (contains the record of all registered voters). The simplest electronic voting design will be a Direct Recording Electronic (DRE) voting system that will embed all these components in a single unit machine.

A more technologically advanced system will be the Public Network Direct Recording Electronic (PNDRE) voting system where the voting terminal and the tallying server sit at remote locations to each other. In this system, a medium of transmitting the ballots from the voting terminal to the tallying server becomes needful. The public network serves the purposes of transmitting the electronic ballots from the voting terminal to the server, normal client/server and also, server/server communications. Most of the current public network design implementations are a VSAT connection with Modem provided by an Internet Service Provider (ISP) except for non supervised electronic voting where voters could cast their votes from any computer with internet connection.

Due to the high cost of acquiring bandwidth, and the fact that acquiring internet connection equipment like VSAT dish, modem and etc. is on the high side, this becomes even more difficult for countries wishing to adopt this technology, as this will amount to procuring this equipment for so many polling stations which will run into thousands of equipment for a country like Nigeria.

This cost can easily be reduced if countries with well developed GSM communication infrastructure can maximize the use of these infrastructures and design electronic voting systems embedded on the GSM technology.

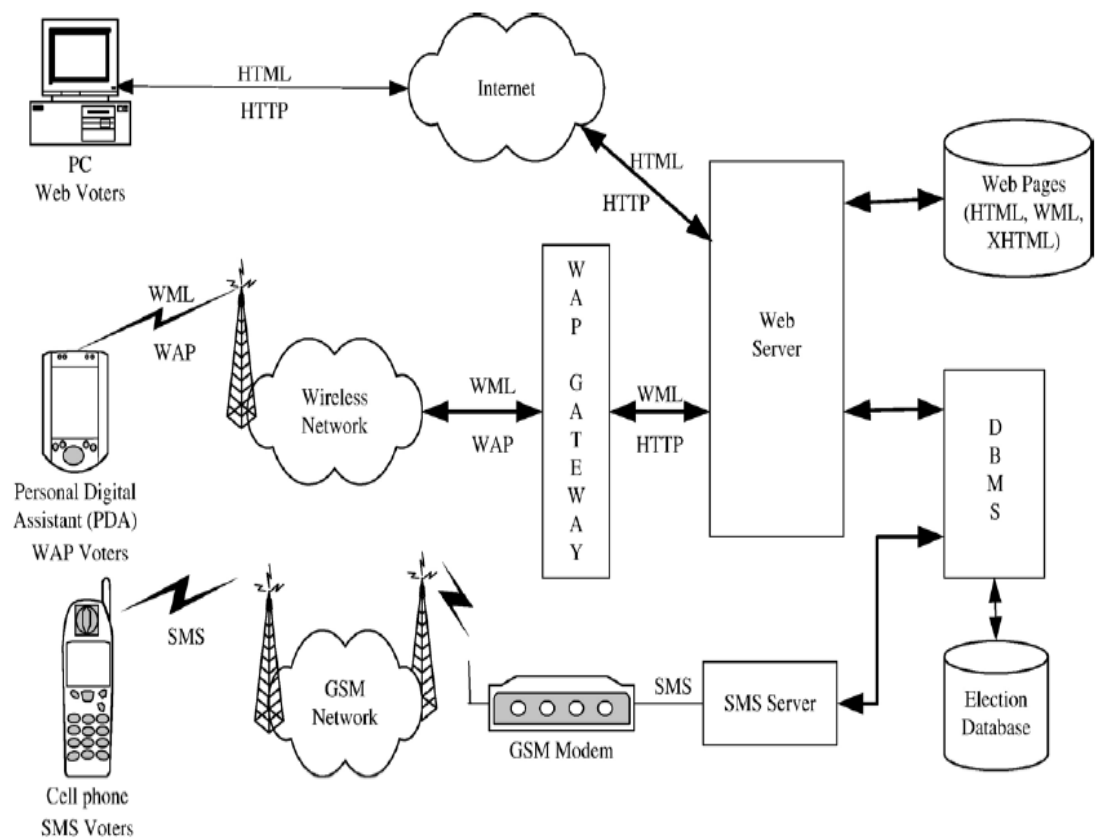

Fig2. Conventional e-voting system architecture

Source: Martine Stenbro (2010).

\section{The Proposed System}

The proposed e-voting design involves transmitting ballots and election information over a secure GSM network. There are three levels on the entire network. These include the public GSM network (where the e-voting terminal nodes reside), the Demilitarized zone (host the proxy server that sits in between the public network and the private network and also hosts the e-voting forwarding server) and the secure Local Area Network (not accessible to nodes on the public network and houses the voter information database and the tallying server). 
Supervised Public Network Direct Recording Electronic Voting (PNDRE Voting) on Existing Global System for Mobile Communication Infrastructure; a Panacea for Cheap E-voting System Implementation in Nigeria

The forwarding server which is hosted in Demilitarized zone (DMZ) is the only server accessible to nodes (e-voting terminals) on the public network. It serves as the intermediary between the public network and the Secure LAN. The voting terminals have to go through the forwarding server to get to either the tallying server or the voter information database. The voting terminals do not know the Internet Protocol address information of servers on the Secure LAN. This information is only known by the forwarding server which performs operations on behalf of the e-voting terminals. This implementation is possible with Dynamic Network Address Translation, where packets are reencapsulated in order to route them to their destination nodes.

The e-voting terminal connects to the network by using a data enabled SIM card provided by the Telecommunication Service providers.

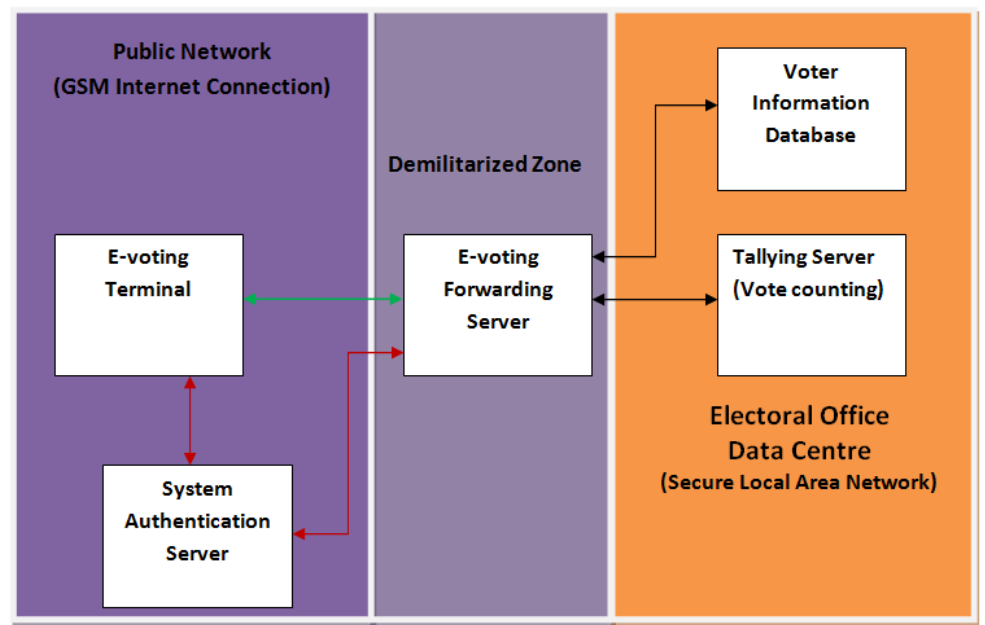

Fig3. Macro model of the proposed e-voting system

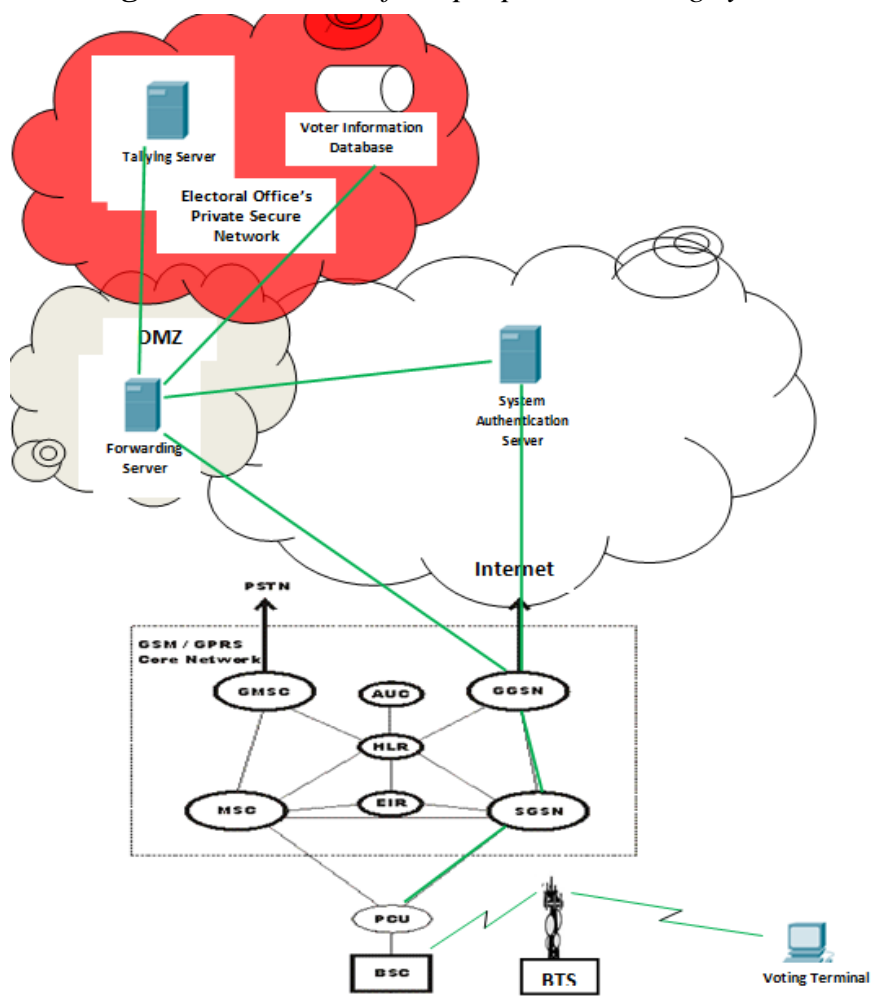

Fig4. Physical architecture of the proposed e-voting system ${ }^{l}$

1 DMZ: Demilitarized Zone, PSTN: Packet Switched Telephone Network, GPRS: General Packet Radio Service, GMSC: Gateway Mobile Switching Centre, AUC: Authentication Centre, GGSN: Gateway GPRS Support Node, HLR: Home Location Register, MSC: Mobile Switching Centre, EIR: Equipment Identity Register, SGSN: Serving GPRS Support Node, PCU: Packet Control Unit, BSC: Base Station Controller, BTS: Base Transceiver Station 


\subsection{New System Operation}

For voting to take place, the voting terminal and the forwarding server have to first authentic to the system authentication server. Keys are distributed between the voting terminal and the forwarding server. These keys will be used to encrypt and decrypt information during communication between these two systems. The preferred protocol for this is asymmetric key cryptography, while the service is provided by a public key infrastructure. According to A.V.N. krishna (2009), Public-key encryption (also called asymmetric encryption) involves a pair of keys, a public key and a private key, used for security \& authentication of data. Each public key is published, and the corresponding private key is kept secret.

After authentication, the voting terminal on behalf of the prospective voter requests for voting privilege from the forwarding server. The forwarding server accepts the requests and forwards the request to the voter information database for confirmation of voter's record on the database. If successful, the forwarding server returns"voter eligible" information to the voting terminal. The terminal in-turn launches the election page for the voter to cast their vote following the necessary procedures.

In order to completely secure the Local Area Network (Secure LAN) from attackers on the public network, a form of cryptography is needed. We propose either a public key cryptography or a symmetric key cryptography. Since this is a private network, implementing public key cryptography will be somewhat costly though, it may be the most effective form of security. Implementing symmetric key cryptography will certainly be cheaper; however one flaw associated with it would be the danger of the shared key being divulged to, or sniffed by an authorised party. The complete operation of this system is beyond the scope of this work. The figure below illustrates the security architecture of the proposed system.

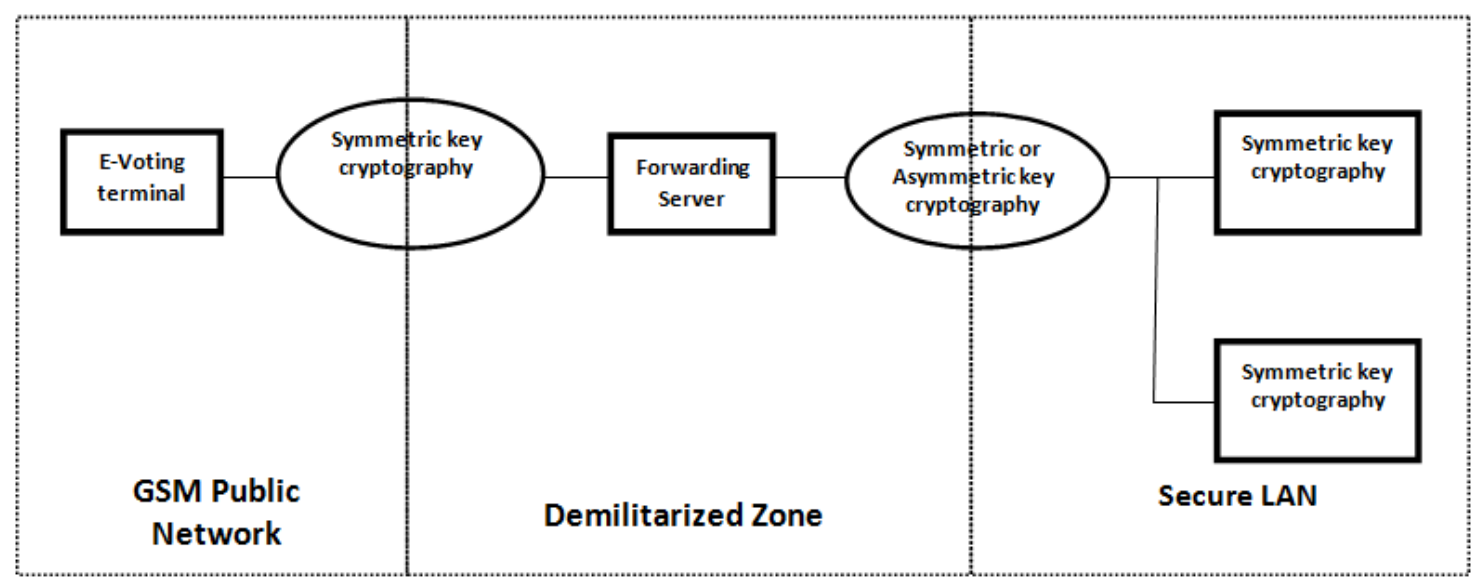

Fig5. Security architecture of the proposed system

\subsection{New System Cost Savings Analysis}

Assuming the total cost of acquiring the e-voting service per polling booth in conventional (VSAT) voting systems is

$\mathrm{w}=\mathrm{x}+\mathrm{y}+\mathrm{z}$

Where:

$\mathrm{w}=$ Total cost of e-voting system per polling booth

$\mathrm{x}=$ Total cost of voting terminal (software + hardware + printer) per polling booth

$\mathrm{y}=$ total cost of logistics (human resource wage, etc.) per polling booth

$\mathrm{z}=$ Total cost of telecommunication service per polling booth

The average relationship between telecommunication service and e-voting system per polling booth as revealed by findings from the survey conducted is;

$\mathrm{z} \geq 0.4 \mathrm{w}$

similarly, $\mathrm{x}+\mathrm{y} \leq \mathrm{w}-\mathrm{z}$ 
Supervised Public Network Direct Recording Electronic Voting (PNDRE Voting) on Existing Global System for Mobile Communication Infrastructure; a Panacea for Cheap E-voting System Implementation in Nigeria

Substituting 2 in 3 results in

$\mathrm{x}+\mathrm{y} \leq \mathrm{w}-0.4 \mathrm{w}$

$\mathrm{x}+\mathrm{y} \leq 0.6 \mathrm{w}$

Implementing our proposed design results in a new relationship

$\mathrm{w}_{\text {new }}=\mathrm{x}_{\text {new }}+\mathrm{y}_{\text {new }}+\mathrm{z}_{\text {new }}$

where,

$\mathrm{w}_{\text {new }}=$ Total cost of e-voting system per polling booth

$\mathrm{x}_{\text {new }}=$ Total cost of voting terminal (software + hardware + printer) per polling booth

$\mathrm{y}_{\text {new }}=$ total cost of logistics (human resource wage, etc.) per polling booth

$\mathrm{Z}_{\text {new }}=$ Total cost of telecommunication service per polling booth

but $\mathrm{z}_{\text {new }}=0$ i.e. For our new design

thus,

$\mathrm{w}_{\text {new }}=\mathrm{x}_{\text {new }}+\mathrm{y}_{\text {new }}$

Assuming $\mathrm{x}_{\text {new }}$ and $\mathrm{y}_{\text {new }}$ remained constant, then

$\mathrm{x}_{\text {new }}+\mathrm{y}_{\text {new }}=\mathrm{x}+\mathrm{y} \leq 0.6 \mathrm{w}$

thus $\mathrm{w}_{\text {new }} \leq 0.6 \mathrm{w}$, thus the cost savings per polling booth $\geq$ forty percent $(40 \%)$

Cost saving per polling booth $=\mathrm{S} \geq 0.4 \mathrm{w}$

Similarly, we can represent the entire cost of acquiring an e-voting system (not per polling booth) with the relationship below

$\mathrm{w}_{\mathrm{t}}=\mathrm{nw}+\mathrm{x}_{\mathrm{t}}+\mathrm{y}_{\mathrm{t}}+\mathrm{z}_{\mathrm{t}}$

where,

$\mathrm{W}_{\mathrm{t}}=$ Total cost of acquiring e-voting system

$\mathrm{w}=$ cost of acquiring e-voting service per polling booth

$\mathrm{x}_{\mathrm{t}}=$ Total cost of other e-voting hardware (non polling booth dependent)

$\mathrm{y}_{\mathrm{t}}=$ Total cost of non polling booth dependent logistics (human resource wage, etc.)

$\mathrm{z}_{\mathrm{t}}=$ Total cost of telecommunication service for the entire system

$\mathrm{n}=1,2 \ldots \ldots \ldots \mathrm{n}=$ Number of polling booths

For our proposed design recall that $\mathrm{w}=\mathrm{w}_{\text {new }}$

Then

$\mathrm{w}_{\mathrm{t}}=\mathrm{nw}_{\text {new }}+\mathrm{x}_{\mathrm{t}}+\mathrm{y}_{\mathrm{t}}+\mathrm{z}_{\mathrm{t}}$

also from our survey, $\mathrm{z}_{\mathrm{t}} \leq 0.05 \mathrm{w}_{\mathrm{t}}$

One can summarily put the cost savings on e-voting implementation using the proposed system as

Total cost saving on e-voting system $=\mathrm{S}_{\mathrm{t}} \geq \mathrm{nS}-\mathrm{z}_{\mathrm{t}}$

substituting 7 and 9 in 10

$\mathrm{S}_{\mathrm{t}} \geq 0.4 \mathrm{nw}-0.05 \mathrm{w}_{\mathrm{t}}$

\section{Conclusion}

Though electronic voting promises great features, flexibility and ease, implementation has continued to be a major challenge to the use of this technology. The opponents of e-voting adoption have continually argued that the technology presents more challenges and disadvantages than solutions to 
its prospective users. This work has done a comprehensive cost analysis of the existing e-voting model together with a new model proposed in this work. This new model suggest a way of bringing down the cost of implementing e-voting in Nigeria by embedding the system only on the existing GSM network infrastructure in the country. This means that countries with low election budgets can as well take advantage of this system to key into the opportunities and ease offered by e-voting if they already have GSM telecommunication infrastructure. The adoption of this model will go a long way in lowering the cost of acquiring an e-voting system in Nigeria, thereby encouraging the implementation of this system even in average to poor countries.

\section{REFERENCES}

Ankit Anand and Pallavi Divya (2012). An Efficient Online Voting System. International Journal of Modern Engineering Research (IJMER), Vol.2, Issue.4, July-Aug. 2012 pp-2631-2634 ISSN: 2249-6645

VoteHere Inc. (2002), Network Voting Systems Standards, Public Draft 2, USA

Oostveen .A.M. and Bessdaar P.V. (2009), Users Experiences with E-voting: A Comparative Case Study, J of Electronic Governance, vol.2, No 4, pp 38-46

Nwogu Emeka Reginald (2015). Mobile, Secure E-Voting Architecture for the Nigerian Electoral System. IOSR Journal of Computer Engineering (IOSR-JCE) e-ISSN: 2278-0661,p-ISSN: 22788727, Volume 17, Issue 2, Ver. II (Mar - Apr. 2015), PP 27-36 www.iosrjournals.org

R. Mercuri (2002). A Better Ballot Box. IEEE Spectrum, Vol.39, No.10, 2002, pp.46-50.

Htet Ne Oo and Aye Moe Aung (2013). Implementation and Analysis of Secure Electronic Voting System. International Journal of Scientific \& Technology Research Volume 2, Issue 3, March 2013 Issn 2277-8616 158 IJSTR@2013 www.ijstr.org

G.O. Ofori-Dwumfuo and E. Paatey (2011). The Design of an Electronic Voting System. Research Journal of Information Technology 3(2): 91-98, 2011 ISSN: 2041-3114

Nigerian Communications Commission (2013). Operator Data. Retrieved $15^{\text {th }}$ July 2015 from http://www.ncc.gov.ng/index.php?option=com_content\&view=article\&id=70:art-statistics operator \&catid $=65 \&$ Itemid $=76$

Nigerian Communication Commission (2014). 2014 Year End Subscriber/ Network Data Report for Telecommunications Operating Companies in Nigeria. Retrieved $15^{\text {th }}$ July 2015 from https://www.google.com.ng/url?sa=t\&rct=j\&q=\&esrc=s\&source=web\&cd=2\&cad=rja\&uact=8\& ved=0CCUQFjABahUKEwjxsp3oqrXHAhUGtRoKHUxVBlY\&url=http\%3A\%2F\%2Fwww.ncc .gov.ng\%2Findex.php\%3Foption\%3Dcom_docman\%26task\%3Ddoc_download\%26gid\%3D656 $\%$ 26Itemid\%3D\&ei=qI_UVfGCIbqasyqmbAF\&usg=AFQjCNEYmYVQY8JZDVxOlOwC8fdB kX0BxA\&sig2=0JVp_yV9bHEijDcVJeTdjQ

Nigerian Tribune (April 7, 2015). Internet Usage on Nigeria's GSM Networks Hit 81 Million. Retrieved $10^{\text {th }}$ June 2015 from http://www.ncc.gov.ng/index.php?option=com_content\&view= article\&id=1626\%3Anigerian-tribune-april-7-2015-internet-usage-on-nigerias-gsm-networks-hit81-million\&catid $=86 \% 3$ Acat-mediapr-headlines $\&$ Itemid $=64$

Martine Stenbro (2010). A Survey of Modern Electronic Voting. Department of Telematics, Norwegian University of Science and Technology. Retrieved $5^{\text {th }}$ June 2015 from https://daim.idi. ntnu.no/masteroppgaver/005/5568/masteroppgave.pdf

A.V.N.Krishna (2009). Performance Evaluation of New Encryption Algorithms with Emphasis on Probabilistic Encryption \& Time Stamp in Network Security. Department of Computer Science and Engineering, Acharya Nagarjuna University, Nagarjuna Nagar - 522510 Andhra Pradesh, India. 